\title{
REPERCUSSÕES DA HEMODIÁLISE NAS ATIVIDADES BÁSICAS E INSTRUMENTAIS DE IDOSOS COM INSUFICIÊNCIA RENAL CRÔNICA
}

\author{
REPERCUSSIONS OF HEMODYALYSIS ON BASIC AND INSTRUMENTAL ACTIVITIES \\ OF ELDERLY WITH CHRONIC RENAL FAILURE
}

\section{RESUMO}

O envelhecimento é processo biopsicossocial, que gera diversas modificações para os indivíduos, principalmente no que tange a capacidade funcional e a Doença Renal Crônica, podendo levar muitos indivíduos para o tratamento hemodialítico. Objetivos: investigar as repercussões da hemodiálise para as atividades básicas e instrumentais da vida diária de idosos em tratamento hemodialítico e descrever as condições associadas. Método: estudo descritivo, quantitativo, de corte transversal, realizado com 35 idosos em tratamento hemodialítico de uma Clínica de Hemodiálise da cidade de Vitória da Conquista - BA. Foram usados três instrumentos, sendo um questionário sobre as condições de saúde e perfil sociodemográfico, a escala Lawton-Brody e o índice de Barthel. A análise foi feita com o software Statistical Package for the Social Sciences. Resultados: prevalência de idosos de 60 a 79 anos (74,3\%), sexo masculino (60\%), casados ou em união estável (60\%). Em tratamento hemodialítico entre 1 a 5 anos (60\%). Dependência parcial para as atividades básicas (100\%), e moderada para as instrumentais $(88,6 \%)$. Nos homens ( $\mathrm{n}=21$ ), quanto maior foi o tempo que tem com a doença renal maior foi a sua limitação para as atividades instrumentais ( 0,000$)$. Nas mulheres $(n=14)$, quanto maior foi o tempo de tratamento, maior foi a sua dependência funcional para as atividades instrumentais ( $\mathrm{p}$ 0,002). Conclusão: o tratamento hemodialítico repercutiu na vida dos idosos, contribuindo para a limitação nas atividades básicas e instrumentais da vida dos mesmos.

\footnotetext{
1 Graduanda em Enfermagem pela Faculdade de Tecnologia e Ciências (FTC). E-mail: patezcamila@gmail.com 2 Graduanda em Enfermagem pela Faculdade de Tecnologia e Ciências (FTC). E-mail: caroul $1 @$ hotmail.com 3 Graduanda em Enfermagem pela Faculdade de Tecnologia e Ciências (FTC). E-mail: biancanovadegraus@gmail.com 4 Graduado em Enfermagem pela Faculdade de Tecnologia e Ciências (FTC). E-mail: cleitonlima.keulima@outlook.com 5 Graduando em Nutrição pela Faculdade de Tecnologia e Ciências (FTC). E-mail: petros2015guardar@gmail.com 6 Doutor em Memória: Linguagem e Sociedade pela Universidade Estadual do Sudoeste da Bahia (UESB). Docente da Faculdade de Tecnologia e Ciências (FTC). E-mail: rnc_novaes@hotmail.com
} 
Descritores: Envelhecimento. Capacidade Funcional. Doença renal crônica. Hemodiálise.

\begin{abstract}
Aging is a biopsychosocial process that generates several changes for individuals, especially regarding functional capacity and chronic kidney disease, and may lead many individuals for hemodialysis treatment. Objectives: To investigate the repercussions of hemodialysis for the basic and instrumental activities of daily living of elderly people undergoing hemodialysis treatment and to describe health conditions associated.. Method: a descriptive, quantitative, cross-sectional study conducted with 35 elderly on hemodialysis treatment of a Hemodialysis Clinic in the city of Vitória da Conquista - BA. Three instruments were used: a questionnaire on health conditions and sociodemographic profile, the Lawton-Brody scale and the Barthel index. The analysis was performed using the Statistical Package for the Social Sciences software. Results: prevalence of elderly aged 60 to 79 years (74.3\%), male (60\%), married or in a stable union (60\%). In hemodialysis treatment between 1 and 5 years (60\%). Partial dependence on basic activities (100\%), and moderate dependence on instrumental (88.6\%). In men ( $n=21)$, the longer their time with kidney disease, the greater their limitation for instrumental activities (p 0.000). In women (n = 14), the longer the treatment time, the greater their functional dependence for instrumental activities ( $p$ 0.002). Conclusion: hemodialysis treatment affected the lives of the elderly, contributing to the limitation of basic and instrumental activities of their lives.
\end{abstract}

Keywords: Aging. Functional capacity. Chronic kidney disease. Hemodialysis.

\title{
INTRODUÇÃO
}

Segundo dados do IBGE (2017), a população brasileira foi estimada em 205,5 milhões de pessoas, sendo que entre 2012 e 2016, a população idosa (com idade igual ou acima de 60 anos) cresceu 16,0\%, chegando a 29,6 milhões de pessoas. Segundo a Organização das Nações Unidas (ONU, 2014) é possível calcular que nas próximas décadas a população mundial com mais de 60 anos, vai atingir a margem de dois bilhões até o ano de 2050.

O envelhecimento é uma resultante da relação entre a idade biológica e a idade cronológica, que envolve uma sequência de alterações fisiológicas, acarretando perda na capacidade funcional em alguns indivíduos. (SILVA ef al., 2015). Esta, por sua vez, pode ser definida como capacidade de o indivíduo conservar habilidades físicas e mentais indispensáveis para um viver independente e autônomo (SANTOS et al., 2015). 
Com a estimativa de vida aumentada surge uma série de consequências e mudanças, visto como um processo biológico, o envelhecimento traz uma diminuição gradual da capacidade funcional, que é progressiva e pode ocasionar as doenças crônicas, que influenciam na capacidade de executar atividades diárias (SANTANA et al., 2017).

A Insuficiência Renal Crônica (IRC) pode ser definida como uma disfunção em que há perda progressiva da função renal e diminuição da taxa de filtração glomerular. Com isso a IRC, também chamada Doença Renal Crônica (DRC), provoca o progressivo acúmulo de toxinas e lixos metabólicos no sangue (GUEVARA et al., 2016).

O indivíduo portador de DRC pode permanecer assintomático até que se tenha $50 \%$ da função renal comprometida. O tratamento pode ser por meio de medicamentos e dieta, e então somente quando a função renal está abaixo de 15 ou $10 \%$ torna-se necessário o uso de métodos invasivos de tratamento, como a diálise que pode ser hemodiálise ou a diálise peritoneal (CARVALHO et al., 2016)

Segundo Carvalho et al (2016) a hemodiálise é a forma de tratamento mais utilizada em idosos com DRC, baseada na circulação extracorpórea. O paciente idoso com DRC em tratamento hemodialítico, necessita conviver com uma doença incurável e de tratamento doloroso, que devido sua evolução e complicações, gera limitações físicas, ansiedades e medos, afetando assim a vida desse indivíduo e de sua família.

Os pacientes com DRC possuem alterações da função física decorrentes da doença, quando submetidos ao tratamento como a hemodiálise, o declínio da função física é intensificado e podemos observar várias alterações tais como: fadiga, câimbras, prostração, anemia e depressão (OLIVEIRA; VIEIRA; BÜNDCHEN, 2018).

Neste contexto, têm-se como objetivos: investigar as repercussões da hemodiálise para as atividades básicas e instrumentais da vida diária de idosos em tratamento hemodialítico e descrever as condições associadas.

A justificativa para a realização do estudo se pauta na necessidade de uma identificação precoce das repercussões que a hemodiálise acarreta para as atividades básicas e instrumentais de idosos. Somado a isso, tem-se neste estudo uma população que merece destaque nas pesquisas, pois os idosos tendem a desenvolver mais a doença devido a diminuição fisiológica da taxa de filtração glomerular, o que se agrava quando há dependência funcional e ao serem submetidos ao tratamento hemodialítico.

A partir daí, pode-se traçar um panorama sobre a relação existente entre a hemodiálise e a dependência funcional do idoso. Os resultados poderão contribuir para ampliação do conhecimento sobre o tema, bem como possibilitará o planejamento de estratégias visando criar incentivos para uma melhor assistência, bem como de políticas públicas para este público. Também evidencia a importância 
pautada na possibilidade de produção de um conhecimento científico que dará subsídios para novas pesquisas nesta abordagem.

\section{REVISÃO BIBLIOGRAFICA}

\section{O PROCESSO DE ENVELHECIMENTO}

Envelhecer é um privilégio, que há muito tempo é desejado pela população, pois antigamente poucas eram as pessoas que conseguiam esse feito. $O$ século XXI trouxe consigo o aumento da população idosa e com isso alguns desafios. (DARDENGO; MAFRA; 2018)

O aumento desta população é um fenômeno mundial, no Brasil entre os anos de 2005 a 2015 houve um aumento da proporção de idosos com idade igual ou superior a 60 anos, passando de 9,8\% para 14,3\% (IBGE, 2016). Ressalta-se que os avanços científicos das ciências em saúde, e as mudanças socioeconômicas têm elevado a média da expectativa de vida ao nascer de 45,5 anos, em 1940, para 74,1 anos, em 2011 (BARBOSA et al., 2014).

O processo de envelhecimento é definido como uma grande mudança fisiológica, essas mudanças são progressivas e engloba diversas alterações, ou seja, é um avanço em desgaste que o corpo sofre com o passar do tempo e tendem a determinar uma acentuada perda da capacidade que o indivíduo possui de se adaptar ao meio ambiente, seja estas dificuldades: físicas, sociais ou psicológicas (OLIVEIRA et al., 2014).

Seguindo nesta perspectiva conceitua o envelhecimento como, um processo que inclui diversas mudanças, sendo elas, políticas econômicas, culturais ou até mesmo os valores, por isso é um processo definido como contínuo. (DARDENGO; MAFRA; 2018).

Envelhecer não significa obrigatoriamente adoecer, exceto quando exista doença associada, o envelhecimento está associado a um bom nível de saúde. Assim cada ser humano envelhece de uma maneira, podendo ou não ter predisposição para adquirir uma patologia (MIRANDA; MENDES; SILVA; 2016).

\section{ENVELHECIMENTO E CAPACIDADE FUNCIONAL}

O envelhecimento é um processo gradativo que como avanço progressivo do tempo pode culminar em diversas perdas físicas, como por exemplo: a capacidade funcional que é entendida como a habilidade física de realizar as atividades normais da vida diária de forma segura e independente. Em indivíduos idosos esse nível funcional já se encontra diminuído e influencia no seu comportamento e na capacidade de executar atividades do dia-a-dia, tais como, subir escadas e carregar objetos (KREUZL; FRANCO, 2017). 
A capacidade funcional dos idosos é avaliada através de uma avaliação funcional que classificavam as atividades cotidianas de acordo com o seu nível de dificuldade abrangendo dois tipos de atividades: Atividades Básicas de Vida Diária (ABVD) e Atividades Instrumentais de Vida Diária (AIVD). A divisão nas categorias ABVD e AIVD começou com os estudos de Mahoney e Barthel e Lawton e Brody (BARBOSA et al., 2014).

\section{ENVELHECIMENTO E HEMODIÁLISE}

Segundo Mendonça et al. (2018) os dados referentes ao envelhecimento trazem também uma preocupação, que é a associação com as Doenças Crônicas Não Transmissíveis (DCNT). Dentre essas patologias, destaca-se a DRC, que atinge pessoas em qualquer faixa etária e sua prevalência vem aumentando significativamente nos últimos anos, caracterizando-a como um grande problema de saúde pública.

Os rins são órgãos essenciais para o equilíbrio da homeostase no corpo humano, a redução progressiva da taxa de filtração glomerular ou a perda das funções renais presentes na DRC provenientes da progressão da idade e dos hábitos no decorrer da vida, comprometem a função biológica do organismo e pode levar o indivíduo a morte de forma rápida (OLIVEIRA et al., 2017).

A DRC se instala de forma lenta e progressiva ocasionando um quadro de falência renal irreversível, entretanto, a progressão pode ser retardada quando diagnosticada e tratada no início (MENDONÇA et al., 2018). A HAS é a comorbidade com maior frequência em indivíduos com enfermidade renal, podendo ser ou não associada com outra patologia; é considerada como um dos principais fatores de risco para DRC, por ser uma doença silenciosa, o que dificulta um diagnóstico precoce (GOMES et al., 2018).

De acordo Oliveira et al. (2017) o advento da hemodiálise acarretou no aumento da sobrevida dos pacientes acometidos por doença renal crônica, porém mesmo sendo um tratamento que devolve ao organismo o equilíbrio da homeostase, é altamente debilitante nos aspectos físicos, emocionais e sociais. Com o envelhecimento associado a uma patologia como a DRC, a qualidade de vida (QV) tende a diminuir. Quando relacionado a tratamentos longos como a hemodiálise, a QV fica mais prejudicada considerando que esses idosos são privados de atividades rotineiras, bem como, ficam sujeitos a mudanças em sua rotina, tais como na dieta e a restrição hídrica. $O$ tratamento hemodialítico costuma ser doloroso e limitador tanto para as ABVD como para AIVD (MENDONÇA et al., 2018). 


\section{METODOLOGIA}

Trata-se de uma pesquisa descritiva, quantitativa, de corte transversal, realizada em uma clínica de hemodiálise privada, mas que também é conveniada aoSistema Único de Saúde (SUS), e está localizada na cidade de Vitória da Conquista - BA. A unidade funciona há mais de 30 anos no mercado, oferecendo o serviço de hemodiálise, exames laboratoriais e acompanhamento com nefrologista. Contam com uma equipe composta por médicos, enfermeiros, técnicos de enfermagem, assistente social, psicóloga e nutricionista.

A média mensal de atendimento é de 280 pessoas, incluindo todas as idades, sendo que as sessões são realizadas em três turnos, sendo um grupo que realiza nas segundas, quartas e sextas- feiras e outro grupo nas terças, quintas e sábados.

Os pacientes são encaminhados através do sistema de regulação do governo do estado, ou através de capitação direta do médico no momento da consulta em consultórios particulares.

Constituíram como participantes da pesquisa 35 idosos selecionados por meio dos critérios de elegibilidade. Sendo eles, de inclusão: ter 60 anos de idade ou mais, com pelo menos um ano em tratamento hemodialítico, pois acredita-se que após esse período os indivíduos consigam reconhecer os efeitos colaterais da hemodiálise e sua influência nas Atividades de Vida Diária (AVD); indivíduos que estavam lúcidos e orientados, em condições de verbalizar para responder aos questionamentos da pesquisa. Foram excluídos os idosos que apresentaram qualquer desconforto ou dor.

A unidade atende uma média mensal de 200 idosos portadores de DRC, dependentes de hemodiálise. E a coleta foi realizada em uma semana do mês de março do ano corrente, somente no turno vespertino, horário disponibilizado pela clínica. Houve dificuldade na coleta de dados devido ao estado de saúde dos idosos, pois muitos estavam debilitados e indispostos no momento da coleta se recusando a participar, e nos horários disponibilizados pela direção o número de idosos que correspondia aos critérios de inclusão foi escasso.

Os instrumentos utilizados para realizar a coleta de dados foram três, sendo o primeiro um questionário, elaborado pelos pesquisadores, sobre as condições de saúde, tempo de tratamento, perfil sociodemográfico e econômico dos idosos submetidos ao tratamento hemodialítico.

O segundo foi a escala de Lawton-Brody, que busca identificar o nível de condição funcional do indivíduo a partir das AIVD; avalia atividades comuns como atender telefone, preparar sua refeição, sair e viajar sozinho dentre outros; o resultado se dá através da soma das respostas e pode se classificar da seguinte maneira: Dependência total com 7 pontos, dependência parcial entre $>7<21$ pontos e independência com 21 pontos (NOGUEIRA, et al.,2017). 
O terceiro instrumento foi a escala de Barthel, que avalia a dependência funcional com base nas ABVD, avalia itens tais como: alimentação, higiene pessoal, vestir-se, controle da bexiga, do intestino, deambulação, subir escadas. A pontuação vai de um a cinco por cada questão e a somatória final de cinco a cinquenta pontos. Seu escore final varia de: dependência total com 10 pontos; dependência severa entre 11 a 30 pontos; dependência moderada entre 31 a 45; ligeira dependência entre 46 a 49 pontos e independência total com 50 pontos (SIMOES; FERREIRA; DOURADO, 2018).

Foi utilizado para análise dos dados o software Statistical Package for the Social Sciences (SPSS) versão 20.0 que permite a realização de cálculos estatísticos básicos e complexos obtendo rápida interpretação dos resultados. Logo após o software, os dados foram interpretados com base na estatística descritiva e inferencial e discutidos a partir da literatura referenciada.

Utilizou-se também o coeficiente de Pearson $(r)$ que é usado para correlacionar variáveis quantitativas, onde o valor de $(r)$ varia de uma direção negativa a uma positiva e o valor numérico indica a força da relação entre as variáveis. Nesse sentido, o coeficiente de r pode variar de -1 a 1 (FIGUEIREDO FILHO; SILVA JÚNIOR, 2009). Ainda para os autores, os valores -1 ou 1 indicam que existe uma correlação perfeita entre as variáveis, no entanto, é salientado que valores acima de 0,5 já indicam correlação forte. O teste de correlação levou em consideração a significância estabelecida pela $p$-value $(p \leq 0,01)$.

A pesquisa respeitou os princípios éticos legais que constam na resolução 466/2012 do Conselho Nacional de Saúde. Foi submetido ao Conselho de Ética em Pesquisas (CEP) com seres humanos da Faculdade Independente do Nordeste (FAINOR) e aprovado pelo número do parecer 3.167.224.

\section{RESULTADOS E DISCUSSÃO}

De posse dos dados obtidos foi traçado o perfil sociodemográfico dos participantes como descrito na Tabela 1, onde se observa que em relação à idade dos 35 participantes da pesquisa 26 (74,3\%) tinham idade entre 60 a 79 anos. Houve predomínio do gênero masculino 21 (60\%), e o estado civil, eram casados ou estão em união estável, 21 (60\%). Na escolaridade, 23 (65,7\%) são alfabetizados. Vivem com renda de um salário mínimo 27 (77,1\%), corresidem com mais duas pessoas 16 $(45,7 \%)$. 
Tabela 1 - Distribuição numérica e percentual do perfil sociodemográfico dos idosos. Vitória da Conquista-BA, 2019.

\begin{tabular}{llcc}
\hline CATEGORIAS & VARIÁVEIS & F.A. & F.R. \\
\hline \multirow{3}{*}{ Idade } & Longevos (80 anos ou mais) & 9 & 25,7 \\
& Idosos (até 79 anos) & 26 & 74,3 \\
& Total & 35 & 100,0 \\
\hline \multirow{3}{*}{ Gênero } & Masculino & 21 & 60,0 \\
& Feminino & 14 & 40,0 \\
& Total & 35 & 100,0 \\
\hline \multirow{3}{*}{ Estado Civil } & Solteiro (a), Divorciado/separado (a) ou Viúvo (a) & 14 & 40,0 \\
& Casado/união estável (a) & 21 & 60,0 \\
& Total & 35 & 100,0 \\
\hline \multirow{4}{*}{ Escolaridade } & Não Alfabetizado & 12 & 34,3 \\
& Alfabetizado & 23 & 65,7 \\
& Total & 35 & $\mathbf{1 0 0 , 0}$ \\
\hline \multirow{3}{*}{ Renda familiar } & Nenhuma renda & 1 & 2,9 \\
& Até 1 salário mínimo* & 27 & 77,1 \\
& De 1 a 3 salários mínimos & 7 & 20,0 \\
& Total & 35 & $\mathbf{1 0 0 , 0}$ \\
\hline \multirow{5}{*}{ Corresidência } & Duas pessoas & 16 & 45,7 \\
& Três pessoas & 12 & 34,3 \\
& Quatro pessoas & 4 & 11,4 \\
& Acima de quatro pessoas & 3 & 8,6 \\
& Total & $\mathbf{3 5}$ & $\mathbf{1 0 0 , 0}$ \\
\hline
\end{tabular}

Fonte: Dados da Pesquisa

F.A. Frequência Absoluta; F.R. Frequência Relativa.

* salário mínimo considerado $\mathrm{R} \$ 937,00$

Sobre as condições de saúde dos idosos, observa-se na Tabela 2 que na categoria sobre o estado geral de saúde, o mesmo foi considerado razoável por $23(65,7 \%)$ dos participantes. Em relação ao tempo que possui a DRC, observou-se que a prevalência foi de 1 a 5 anos em 21 (60\%). O tempo em que o idoso está em tratamento hemodialítico foi de 1 a 5 anos em 25 (71,4\%).

Ainda na Tabela 2, as outras patologias referidas pelos participantes estavam centradas nas DCNT, tais como Hipertensão Arterial Sistêmica (HAS) 11(31,4\%) e Diabetes Mellitus (DM) associada à HAS também em 11 (31,4\%). Em relação ao tempo em que o idoso possui essa patologia associada, a prevalência foi de dez anos em $17(48,6 \%)$ dos entrevistados. Sobre o consumo de cigarro e bebida alcoólica, todos os participantes relataram nunca ter exercido essa prática. 
Tabela 2 - Distribuição numérica e percentual das condições de saúde dos idosos. Vitória da Conquista-BA, 2019.

\begin{tabular}{|c|c|c|c|}
\hline CATEGORIAS & VARIÁVEIS & F.A. & F.R. \\
\hline \multirow{4}{*}{ Estado Geral de saúde } & Bom & 11 & 31,4 \\
\hline & Razoável & 23 & 65,7 \\
\hline & Mal & 1 & 2,9 \\
\hline & Total & 35 & 100,0 \\
\hline \multirow{4}{*}{ Tempo que o idoso possui doença renal } & De 1 a 5 anos & 21 & 60,0 \\
\hline & De 6 a 9 anos & 8 & 22,9 \\
\hline & De 10 anos acima & 6 & 17,1 \\
\hline & Total & 35 & 100,0 \\
\hline \multirow{4}{*}{ Tempo que idoso faz hemodiálise } & De 1 a 5 anos & 25 & 71,4 \\
\hline & De 6 a 9 anos & 8 & 22,9 \\
\hline & De 10 anos acima & 2 & 5,7 \\
\hline & Total & 35 & 100,0 \\
\hline \multirow{7}{*}{ Outras patologias relatadas } & HAS & 11 & 31,4 \\
\hline & DM & 5 & 14,3 \\
\hline & $\mathrm{HAS} / \mathrm{DM}$ & 11 & 31,4 \\
\hline & HAS / DC & 6 & 17,1 \\
\hline & HAS / DR & 1 & 2,9 \\
\hline & COLUNA & 1 & 2,9 \\
\hline & Total & 35 & 100,0 \\
\hline \multirow{4}{*}{ Tempo com a outra patologia } & De 1 a 5 anos & 7 & 20,0 \\
\hline & De 6 a 10 anos & 11 & 31,4 \\
\hline & Acima de 10 anos & 17 & 48,6 \\
\hline & Total & 35 & 100,0 \\
\hline
\end{tabular}

Fonte: Dados da Pesquisa

F.A. Frequência Absoluta; F.R. Frequência Relativa; HAS - Hipertensão Arterial Sistêmica; DM - Diabetes Mellitus; DC - Doença Cardíaca; DR - Doença Respiratória.

A tabela 3 abaixo aborda sobre a dependência funcional dos idosos, onde é possível observar na categoria ABVD avaliada através da escala de Barthel, que a dependência parcial teve uma maior frequência, sendo de 35 (100\%). E na categoria AIVD, avaliada através da escala de Lawton e Brody, houve maior prevalência de dependência moderada, sendo no total de 31 (88,6\%).

Tabela 3 - Distribuição percentual e numérica da classificação da dependência funcional dos idosos Vitória da Conquista - BA, 2019.

\begin{tabular}{llcc}
\hline CATEGORIAS & VARIÁVEIS & F.A. & F.R. \\
\hline Barthel (ABVD) & Dependência Parcial & 35 & 100,0 \\
\hline \multirow{3}{*}{ Lawton e Brody (AIVD) } & Ligeiramente Dependente & 4 & 11,4 \\
& Dependência Moderada & 31 & 88,6 \\
& Total & 35 & 100,0 \\
\hline
\end{tabular}

Fonte: Dados da Pesquisa

F.A. Frequência Absoluta; F.R. Frequência Relativa. 
Os resultados na Tabela 4 indicam que com relação ao gênero masculino, houve correlação de Pearson significativa e positiva (r 1,000) nas variáveis "Tempo que o idoso possui doença renal" e "DF para AIVD", ou seja, para os homens pesquisados ( $\mathrm{n}=21$ ) quanto maior foi o tempo que ele tem com a doença renal maior foi a sua limitação física para as AIVD ( $p$ 0,000).

Também com o gênero feminino a correlação foi significativa e positiva ( $r$ $0,685)$ para as variáveis "Tempo que idoso faz hemodiálise" com "DF para AIVD", sendo que para elas ( $n=14$ ) quanto maior foi o tempo em que elas estão fazendo hemodiálise maior foi a sua dependência funcional para as AIVD (p 0,002).

Tabela 4 - Correlação entre gênero e tempo de doença renal e de hemodiálise com AIVD. Vitória da Conquista - BA, 2019.

\begin{tabular}{|c|c|c|c|c|}
\hline Categoria & Variável & Variável & DF & \\
\hline \multirow{6}{*}{ Gênero } & \multirow{3}{*}{ Masc. } & \multirow{3}{*}{$\begin{array}{l}\text { Tempo que o idoso possui } \\
\text { doença renal }\end{array}$} & Pearson (r) & $1,000 *$ \\
\hline & & & $\mathrm{N}$ & 21 \\
\hline & & & p-value & $0,000 * *$ \\
\hline & \multirow{3}{*}{ Fem. } & \multirow{3}{*}{$\begin{array}{l}\text { Tempo que idoso faz } \\
\text { hemodiálise }\end{array}$} & Pearson (r) & $0,685^{*}$ \\
\hline & & & N & 14 \\
\hline & & & p-value & $0,002 * *$ \\
\hline
\end{tabular}

Fonte: Dados da pesquisa.

Masc - Masculino. Fem - Feminino. n - Amostra. DF - Dependência Funcional

*Correlação significativa $>0,5$. ${ }^{*}$ Considerar significância valor de $p$-value $<0,01$

\section{DISCUSSÃO}

De acordo o perfil traçado, as características encontradas corroboram o estudo de Santos et al. (2017), realizado em um município do Piauí, com 172 participantes, sobre a qualidade de vida de pacientes com IRC sob tratamento hemodialítico. Nesta ocasião, os autores comprovaram que $67,9 \%$ foi do sexo masculino. Também de acordo Sesso et al. (2017), no ano de 2016 no Brasil, o número de pacientes do sexo masculino com DRC em tratamento hemodialítico representava $57 \%$ do total, e 21,8\% possuíam a idade entre 65 e 74 anos, ratificando os achados deste estudo.

A senescência ${ }^{7}$ gera uma maior vulnerabilidade de se apresentar DCNT, seja decorrente da fragilidade ocasionada pela própria idade e as comorbidades, seja devido aos fatores externos associados, como a baixa renda e baixa escolaridade destes idosos, que acabam por gerar a limitação a procura do acesso ao serviço de saúde e o entendimento das complicações da doença (SCHUCK; ANTONI, 2018).

7 Processo natural do envelhecimento humano. Somatória de alterações orgânicas, funcionais, psicológicas próprias do envelhecimento normal. Fonte: http://www.sbgg-sp.com.br/pub/senescencia-e-senilidade-qual-a-diferenca/. Sociedade Brasileira de Geriatria e Gerontologia (2016). 
As condições socioeconômicas dos idosos com DRC em tratamento hemodialítico são fatores muito importantes e devem ser considerados, pois deles dependerão, na maioria das vezes, a continuidade do tratamento, e todas as demais despesas da família. Outro fator é o número de pessoas dependentes da renda dos idosos, pois isso poderá influenciar no acesso deles às condições de saúde. Sendo assim, o idoso com uma condição crônica, como a DRC, dependente de um tratamento hemodialítico, podem ser considerados fatores que o impedem de desenvolver alguma atividade laboral, para proporcionar um aumento em sua renda, consequentemente refletindo nas suas condições de saúde (BOSENBECKER et al., 2015).

Observado o perfil das condições de saúde dos idosos, é possível inferir que de acordo Meira et al. (2016), a DRC influência nas alterações metabólicas e na perda da reserva funcional, acelerando o processo de envelhecimento. Isso explica a dependência e fragilidade no dia a dia de pacientes em tratamento hemodialítico, o tempo de tratamento assim como o tempo que possui a DRC compromete a sua condição de saúde, ocasionando dependência funcional. De acordo Oliveira et al. (2017) o advento da hemodiálise acarretou no aumento da sobrevida dos pacientes acometidos por DRC, porém mesmo sendo um tratamento que devolve ao organismo o equilíbrio da homeostase, é altamente debilitante nos aspectos físicos, emocionais e sociais.

O acometimento por DCNT é comumente observada em pessoas idosas e é um fator de grande relevância para o desenvolvimento de incapacidade funcional, e a autonomia desses portadores pode se encontrar limitada (ABREU et al., 2017). O aumento da DRC está estreitamente relacionado à ocorrência de DCNT, em especial a DM e HAS que estão se tornando mais frequentes na população em geral (MENDONÇA et al., 2018).

Oenvelhecimento é um processo gradativo que como avanço progressivo do tempo pode culminar em diversas perdas físicas, como por exemplo: a capacidade funcional que é entendida como a habilidade física de realizar as atividades normais da vida diária de forma segura e independente. Em indivíduos idosos esse nível funcional já se encontra diminuído e influencia no seu comportamento e na capacidade de executar atividades do dia-a-dia, tais como, subir escadas e carregar objetos (KREUZL; FRANCO, 2017).

O impacto da DRC referente a dificuldade em realizar as ABVD, contribuem para diminuição da QV, devido às repercussões musculoesqueléticas comuns em pacientes submetidos à hemodiálise. Ocasionando nesses idosos uma queda no desempenho funcional e no desempenho das atividades (SANTOS; SARDINHA, 2018).

De acordo Jesus et al. (2019), o tratamento hemodialítico causa considerável comprometimento funcional e físico no indivíduo, sendo comum o aparecimento de problemas como sedentarismo, diminuição da interação social, perda da autonomia e dependência, pois o mesmo passa a necessitar de ajuda de outras 
pessoas para realizar várias atividades do dia a dia. Ainda para os autores, o indivíduo com DRC encontra grande dificuldade em estabelecer e/ou manter um vínculo de trabalho devido ao tempo dedicado e a rotina exigida pelo tratamento que associado aos sintomas físicos e emocionais interferem nas atividades diárias e nos aspectos psicoemocionais.

O estudo ora exposto demonstra que há correlação entre o tempo que o idoso tem a doença e a dependência funcional para AIVD. Para as mulheres o tempo que fazem hemodiálise influencia a dependência funcional para AIVD. Nesse sentido, pode-se inferir que tanto o tempo de doença como o tempo que faz hemodiálise geram repercussões para as AIVD de homens e mulheres idosos. Nesse sentido, de acordo Gomes et al. (2018), o indivíduo que está em tratamento hemodialítico apresenta implicações em inúmeros aspectos referentes à saúde física e mental, sua capacidade funcional e independência, gerando limitações para realizar as AVD.

Dessa forma, ao analisar as diferentes repercussões que a hemodiálise acarreta para homens e mulheres, é possível perceber que a DRC, assim como o processo diário de hemodiálise geram diferentes impactos na rotina de homens e mulheres. Assim, Gomes et al. (2018), salientam que em indivíduos do sexo masculino, a vulnerabilidade resultante do processo da DRC e a necessidade de cuidados constantes favorecem o aparecimento de sentimentos negativos que influenciam em suas ABVD.

Para a mulher, a DRC assim como o tratamento gera diversos tipos de sentimentos e dúvidas quanto ao futuro e ao cuidado familiar surgem com mais veemência. Essas alterações dos papeis vivenciada por ela provocam mudanças nos hábitos familiares, pois muitas vezes a mesma passa de cuidadora principal à pessoa cuidada (OLIVEIRA et al., 2016)

No mesmo sentido, salientam Santos et al. (2017), que a experiência do tratamento hemodialítico e a dependência da máquina podem gerar sofrimento e aflição, pois a hemodiálise é um tratamento que traz muita dor, uma rotina monótona e limitada. O indivíduo que faz uso desse tratamento é submetido a perdas significativas na saúde e no vigor físico, manifestados pela apresentação de sintomas como sonolência, sensação de mal-estar, pressão cefálica e fadiga durante a realização da hemodiálise. Ainda para os autores, conviver com a perda da liberdade e da capacidade produtiva para as atividades também são algumas das realidades vivenciadas pelos pacientes com DRC.

\section{CONSIDERAÇÕES FINAIS}

Os resultados apresentados revelaram que o tratamento hemodialítico mesmo sendo o mais convencional repercutiu na vida dos idosos participantes da 
pesquisa, uma vez que trouxe limitações, e contribuiu para a limitação nas atividades básicas e instrumentais da vida dos idosos.

Nesse sentido, a hemodiálise traz inúmeras repercussões na vida destes idosos, pois uma vez diagnosticados, terão que lidar com novo estilo de vida, que por consequência traz várias mudanças. Sendo assim os resultados evidenciam prevalência de dependência paras as ABVD e AIVD.

Com relação às condições de saúde, a pesquisa demonstrou que os idosos consideram seu estado geral de saúde como razoável, sobre o tempo de tratamento a prevalência foi de 1 a 5 anos e o perfil sociodemográfico demonstra que são em sua maioria (até 79 anos), do gênero masculino, casados ou em união estável, alfabetizados, com renda familiar de até um salário mínimo e corresidem com até duas pessoas. E na classificação da dependência funcional, foi observado que apresentam dependência parcial para as ABVD, observados pela escala de Barthel e dependência moderada para as AIVD, avaliada através da escala de Lawton e Brody.

Sobre as limitações do estudo, estes envolveram a disponibilidade do local da pesquisa apenas em um turno, bem como o fato dos idosos estarem indispostos e desconfortáveis com o próprio tratamento, fazendo com que alguns se recusassem a participar das pesquisas.

No entanto, com base nos achados, é importante que tanto familiares, como os profissionais se atentem à essas necessidades dos idosos em realizar as atividades básicas e instrumentais da vida diária, visando prestar um cuidado que englobe o pós tratamento hemodialítico, afim de que os impactos causados pela hemodiálise sejam diminuídos, garantindo a qualidade de vida e mantendo a capacidade funcional desses idosos.

\section{REFERÊNCIAS}

ABREU, S.S.S.; OLIVEIRA, A.G.; MACEDO, M.A.S.S.; DUARTE, S.F.P.; REIS, L.A.; LIMA, P.V. Prevalência de doenças crônicas não transmissíveis em idosos de uma cidade do interior da Bahia. Revista multidisciplinar e de psicologia. Cariri-CE.v.1 1, n.38, p. 652662, 2017. Disponível em: https://idonline.emnuvens.com.br/id/article/view/963/1367. Acesso em 22 de Maio de 2019.

BARBOSA, B.R.; ALMEIDA, J.M.; BARBOSA, R.M.; BARBOSA, L.A.R.R. Avaliação da capacidade funcional dos idosos e fatores associados à incapacidade. Revista Ciência e Saúde Coletiva. Rio de Janeiro - RJ. v19, n 8, p. 3317 - 3325. 2014.Disponível em: https://www.scielosp.org/article/csc/2014.v19n8/3317-3325/pt/ . Acesso em 28 de Maio de 2018. 
BOSENBECKER, N.R.V.; MENEGON, M.B.C.; ZILLMER, J.G.V.; AGNOL, J.D. Perfil das pessoas em hemodiálise de um serviço de nefrologia. Journal of Nursing and Health - Faculdade de Enfermagem UfPEL. Pelotas - RS.v.5, n.1, p. 38-46, 2015. Disponível em: https://periodicos.ufpel.edu.br/ojs2/index.php/enfermagem/article/ view/5337/4299. Acesso em 15 de Abril de 2019.

CARVALHO, F.P.; CARVALHO, I.L.N.; SOUSA, A.S.J.; SIMÕES, C.D.; SILVA, E.S.; SANTOS, J.A.F. Avaliação da capacidade funcional de idosos com doença renal crônica em tratamento de hemodiálise. Revista Saúde - Santa Maria. v.42, n.2, p. 175-184, 2016. Disponível em: https://periodicos.ufsm.br/revistasaude/article/view/21515. Acesso em 20 de Abril de 2018.

DARDENGO, C.F.R.; MAFRA, S.C.T. Os conceitos de velhice e envelhecimento ao longo do tempo: contradição ou adaptação?. Revista de Ciências Humanas, Viçosa, MG, n. 2, out. 2019. ISSN 2236-5176. Disponível em: https://periodicos.ufv.br/ RCH/article/view/8923. Acesso em: 21 Novembro de 2019.

FIGUEREDO FILHO, D.B.; SILVA JÚNIOR, J. A. Desvendando os Mistérios do Coeficiente de Correlação de Pearson (r). Universidade Federal de Pernambuco. Revista Política Hoje. Recife-PE. Vol.18, n1, 2009.

GOMES, N.D.B.; LEAL, N.P.R.; PIMENTA, C.J.L.; MARTINS, K.P.; FERREIRA, G.R.S.; COSTA, K.N.F.M. Qualidade de vida de homens e mulheres em hemodiálise. Revista Baiana de Enfermagem. João Pessoa - PB. v.32, e.24935, p.1-10, 2018. Disponível em: https:// rigs.ufba.br/index.php/enfermagem/article/view/24935/16480. Acesso em 22 de maio de 2019.

GUEVARA, D. E. D.; OLIVEIRA, R.E.N.N.; DUARTE, R.S.; RODRIGUES, A. M. Qualidade de vida de pacientes portadores de insuficiência renal crônica submetidos a hemodiálise em Cacoal-Ro. Revista eletrônica facimedit. Cacoal - RO. v.5, n.2, p. 1-12, 2016. Disponível em: http://repositorio.facimed.edu.br/xmlui/bitstream/ handle/123456789/54/Guevara.pdf? sequence=1\&isAllowed=y. Acesso em 19 de Maio de 2019.

IBGE. PNAD 2016: População idosa cresce 16,0\% frente a 2012 e chega a 29,6 milhões. Agencia IBGE Notícias. 2017. Disponível em: https://agenciadenoticias.ibge.gov.br/ agencia-noticias/2013-agencia-denoticias/releases/18263-pnad-2016-populacaoidosa-cresce-16-0-frente-a-2012-echega-a-29-6-milhoes.html. Acesso em 20 de Abril de 2018. 
IBGE. Sis 2016: 67,7\% dos idosos ocupados começaram a trabalhar com até 14 anos. CENSO 2010. 2016. Disponível em: https://censo2010.ibge.gov.br/noticiascenso. $\mathrm{html}$ ? busc $a=1$ \& id= 1 \&idnoticia=3326\&t=sis-2016-67-7-idosos-ocupadoscomecaramtrabalhar-14-anos\&view=noticia. Acesso em 05 de Junho de 2018.

JESUS, N.M.; SOUZA, G.F.; RODRIGUES, C.M.; NETO, O.P.A.; RODRIGUES, D.M. CUNHA, C.M. Qualidade de vida de indivíduos com doença renal crônica em tratamento dialítico. Braz. J. Nephrol. (J. Bras. Nefrol.). São Paulo - SP. p. 1- 11, 2019. Disponível em: $\quad$ http://www.scielo.br/pdf/jbn/2019nahead/pt_2175-8239-jbn-2018-0152.pdf. Acesso em 10 de junho de 2018.

KREUZ, G; FRANCO, M..H.P. O luto do idoso diante das perdas da doença e do envelhecimento - Revisão Sistemática de Literatura. Arquivo Brasileiro de Psicologia. Rio de Janeiro - RJ. v. 69, n. 2, p.168-186, 2017. Disponível em: http://pepsic.bvsalud. org/pdf/arbp/v69n2/12.pdf. Acesso em 01 de maio 2018.

MARCONI; Marina; LAKATOS, Maria. Métodos e técnicas de pesquisa social. São Paulo, Atlas, 2010.

MEIRA, A.S.; BATISTA, M.A.; PEREIRA, R.M.P; RODRIGUES, R.A.P.; FHON, J.R.S.; KUSUMOTA, L. Fragilidade em idosos com doença renal crônica em tratamento conservador. Revista Rene. Ceará. v.17, n.3, p. 386 - 392, 2016. Disponível em: http:// www.periodicos.ufc.br/rene/article/view/3475/2718. Acesso em 24 de Abril de 2019.

MENDONÇA, A.E.O.; JÚNIOR, B.S.S.; DANTAS, J.G.; ANDRANDE, D.A.; SEGATO, C.T.; VALENÇA, C.N. Adesão de idosos com insuficiência renal crônica a terapia hemodialítica. Revista de enfermagem USFM. Santa Maria- RS. v.8, n.1, p. 49 - 54, 2018. Disponível em: file://C:/Users/WInfor/Downloads/25353-153380-1-PB\%20(1). pdf. Acesso em 16 de maio de 2019.

MIRANDA, G.M.D; MENDES, A. C.G; SILVA, A. L. A. O envelhecimento populacional brasileiro: desafios e consequências sociais atuais e futuras. Revista Brasileira de Geriatria e Gerontologia. Rio de Janeiro. v.19, n.3, p. 507-519, 2016. Disponível em: http://www.redalyc.org/articulo.oa?id=403846785012. Acesso em 24 de Março de 2019.

NOGUEIRA, P. S.F.; MARQUES, M.B.; COUTINHO, J.F.V.; MAIA, J.C.; SILVA, M.J.; MOURA, E.R.F. Fatores associados à capacidade funcional de idosos com hanseníase. Revista Brasileira de Enfermagem. Brasília. v.70, n.4, p. 744-51. 2017. Disponível em https:// www.redalyc.org/pdf/2670/267052023007.pdf. Acesso em 23 de setembro de 2019. 
OLIVEIRA, A.C.F; VIEIRA, D.S.R; BUNDCHEN, D.C. Nível de atividade física e capacidade funcional de pacientes com doença renal crônica pré-dialítica e em hemodiálise. Revista Fisioterapia e Pesquisa. São Paulo. v.25, n. 3, p. 323-329, 2018. Disponível em: http://www.scielo.br/pdf/fp/v25n3/2316-9117-fp-25-03-323.pdf. Acesso em 21 de maio de 2019.

OLIVEIRA, N. B. B.; ALBUQUERQUE, J.M.M.; TAVARES, I.M.; FREITAS, M.A.A.F.; COSTA, M.V. Insuficiência renal crônica: o impacto da hemodiálise na qualidade de vida do idoso. Congresso Internacional de Envelhecimento Humano. 2017. Disponível em: http://www.editorarealize.com.br/revistas/cieh/trabalhos/TRABALHO_EV075_MD4_S A3_ID2951_23102017230931.pdf. Acesso em 03 de maio de 2018.

OLIVEIRA, N.S.; SOUZA, T.S.; ALENCAR, F.S.; OLIVEIRA, G.L.; FERREIRA, N.B.; ALENCAR, J.S. Percepção dos idosos sobre o processo de envelhecimento. Revista de psicologia. Iguatu-CE. v. 8, n.22, p. 1-35, 2014. Disponível em: https://idonline.emnuvens.com.br/ $\mathrm{id} /$ article/view/264/376. Acesso em 02 de Maio de 2018.

OLIVEIRA, V.A.; SCHWARTZ, E.; SOARES, M.C.; SANTOS, B.P.; GARCIA, R. P.; LISE, F. Relações familiares de mulheres em hemodiálise. Revista de Atenção à Saúde. São Caetano do sul- São Paulo. v. 14, n. 47, p. 36-42, 2016.Disponivel em: http://seer.uscs. edu.br/index.php/revista_ciencias_saude/article/view/3283/pdf. Acesso em 31 de maio de 2019.

ONU. Mundo terá 2 bilhões de idosos em 2050; OMS diz que "envelhecer bem deve ser prioridade global". 2014. Disponível em: https://nacoesunidas.org/ mundotera-2-bilhoes-de-idosos-em-2050-oms-diz-que-envelhecer-bem-deve-serprioridadeglobal/. Acesso em 20 de Abril de 2018.

POLIT. D.F.; BECK.C.T. HUNGLER. B.P. Fundamentos de Pesquisa em Enfermagem. 5ed. Porto Alegre: Artmed, 2004.

SANTANA, E. S.; CHAVES, R.N.; LIMA, P.V.; VALENÇA, T.D.C.; REIS, L.A. Limites do envelhecer: percepção de idosos com dependência funcional no interior da Bahia. Revista Uniabeu. Rio de Janeiro. v. 10, n. 24, p. 206-219, 2017. Disponível em: https:// revista.uniabeu.edu.br/index.php/RU/article/view/2529. Acesso em 30 de Abril de 2018.

SANTOS, A.C.B.C.; SIQUEIRA, J.A.S.; NASCIMENTO, I.N.A.; LIMA, F.V. Alterações na capacidade funcional em mulheres na menopausa. Revista InterScientia. v.3, n.2, p. 123-132, 2015. Disponível em: https://periodicos.unipe.br/index.php/interscientia/ article/view/18/15. Acesso em 24 de Agosto de 2019. 
SANTOS, R.R; FORMIGA, L.M.F; OLIVEIRA, E.A.R; LIMA, L.H.O; ARAÚJO, A.K.S; BRITO, B.B. Qualidade de vida de pacientes com insuficiência renal crônica sob tratamento hemodialítico. Revista Interdisciplinar. Itabaiana - SE. v. 8, n. 3, p. 83-92, 2015. Disponível em: https://revistainterdisciplinar.uninovafapi.edu.br/index.php/revinter/ article/view/715/pd f_238. Acesso em 17 de maio de 2019.

SANTOS, R.S.S; SARDINHA, A.H.L. Qualidade de vida de pacientes com doença renal crônica. Enfermagem em foco. Salvador - Ba.v.9, n.2, p. 61-66, 2018. Disponível em: http://revista.cofen.gov.br/index.php/enfermagem/article/view/1078/447. Acesso em 17 de Maio de 2019.

SANTOS, B.P.; OLIVEIRA, V.A.; SOARES, M.C.; SCHWARTZ, E. Doença renal crônica: relação dos pacientes com a hemodiálise. Revista Brasileira - Arquivo brasileiro de ciência da saúde. Sandro André- São Paulo v.42,n.1,p.8-14,2017. Disponível em: https://www.portalnepas.org.br/abcshs/article/view/943/755. Acesso em 31 de Maio de 2019.

SCHUCK, L.M; ANTONI, C. Resiliência e Vulnerabilidade nos sistemas ecológicos: Envelhecimento e políticas públicas. Psicologia Teoria e Pesquisa. Brasília. v. 34, e.3442, p. 1-9, 2018.Disponível em: http://www.scielo.br/scielo.php?script=sci_ arttext\&pid=S0102-37722018000100501\&lng=pt\&tlng=pt. Acesso em 18 de março de 2019.

SESSO, R.C.; LOPES, A.A.; THOMÉ, F.S.; LUGON, J.R.; MARTINS, C.T. Inquérito Brasileiro de Diálise Crônica 2016. Jornal Brasileiro de Nefrologia. São Paulo - SP.v.39, n.3, p. 261-266, 2017.Disponível em: http://www.scielo.br/pdf/jbn/v39n3/pt_0101-2800jbn39-03-0261.pdf. Acesso em 25 de Abril de 2018.

SILVA, P.A.; SILVA, K.O.; MASCARENHAS, G.D.M.; FARIA, L.A. Aspectos relevantes da farmacoterapia do idoso e os fármacos inadequados. Revista InterScientia. João Pessoa - PB. v.3, n.1 , p.31-47, 2015. Disponível em: https://periodicos.unipe.br/index. php/interscientia/article/view/95/91. Acesso em 24 de Agosto de 2019.

SIMOES, A.L.; FERREIRA, P.L.; DOURADO, M. Medição da autonomia em atividades da vida diária. Portuguese Journal Of Public Health. Lisboa-PO. v. 36, n. 1, p. 1-7, 2018. Disponível em: http://www.scielo.mec.pt/pdf/pjph/v36nl/v36nla03.pdf. Acesso em 20 de setembro 2019. 